\title{
Comparator-based switched-capacitor pipelined analog-to-digital converter with comparator preset, and comparator delay compensation
}

\author{
Carsten Wulff • Trond Ytterdal
}

Received: 13 March 2010/Revised: 30 November 2010/Accepted: 3 December 2010/Published online: 19 December 2010

(C) The Author(s) 2010. This article is published with open access at Springerlink.com

\begin{abstract}
We present a differential comparator-based switched-capacitor (CBSC) pipelined analog-to-digital converter (ADC) with comparator preset, and comparator delay compensation. Compensating for the comparator delay by digitally adjusting the comparator threshold improves the ADC resolution from 2.5-bit to 7.05-bit. The ADC is manufactured in a $90 \mathrm{~nm}$ CMOS technology, with a core area of $0.85 \mathrm{~mm} \times 0.35 \mathrm{~mm}$, a $1.2 \mathrm{~V}$ supply for the core and $1.8 \mathrm{~V}$ for the input switches. It has an effective number of bits (ENOB) of 7.05-bit, and a power dissipation of $8.5 \mathrm{~mW}$ at $60 \mathrm{MS} / \mathrm{s}$.
\end{abstract}

Keywords Analog-to-digital converter - Comparatorbased switched-capacitor circuit

\section{Introduction}

Scaling down CMOS technology into the nano-scale range (below $100 \mathrm{~nm}$ gate-length) creates new challenges for the analog designer. Gone are the days of transistors with high output resistance and high voltage tolerance. With a power supply of $1.2 \mathrm{~V}$ in a $90 \mathrm{~nm}$ CMOS technology, and a threshold voltage up to $0.5 \mathrm{~V}$ in a low-power flavor there is not much headroom to stack transistors, and when the transistor intrinsic gain ${ }^{1}$ worsens with each technology node-down to a gain of around 16 times in $90 \mathrm{~nm}$ - it makes a high performance operational transconductance amplifier (OTA) harder to design. This presents a challenge because OTAs are the key component in most

C. Wulff $(\bowtie) \cdot$ T. Ytterdal

Norwegian University of Science and Technology,

Trondheim, Norway

e-mail: carsten@wulff.no switched-capacitor circuits, and switched-capacitor circuits are the de facto standard for implementing ADCs. The challenges in nano-scale CMOS has caused a flurry of research into removing the OTA, where one of the research avenues has led to the comparator-based switched-capacitor circuits (CBSC) $[1,2]$. In this paper we'll present a pipelined ADC based on this technique.

This paper is organized as follows, we'll introduce comparator-based switched-capacitor circuits in Sect. 2. Sections 3 and 4 describe in detail the implementation of the ADC. The test setup is detailed in Sect. 5 and the measurement results explained in Sect 6.

\section{Comparator-based switched-capacitor circuits}

A nice thing about the switched-capacitor circuit is that it does not matter how it arrives at the output voltage. We just have to make sure that the output voltage is correct when the next stage samples. Back in 2006 an idea was put forth for a new method of implementing switched-capacitor circuits [1]. Instead of an OTA, they used a current source and a comparator, and called it CBSC, that name has later transformed into zero-crossing-based switched-capacitor circuits (ZBSC), but we'll use the original name, CBSC, in this paper, because this work was based on the original publication.

An example of a single-ended CBSC amplifier in the charge transfer phase is shown in Fig. 1. In the sampling phase (not shown) the input voltage is sampled across both capacitors. At the start of the charge transfer phase the output is reset to the lowest voltage in the system. This

\footnotetext{
1 The intrinsic gain is the transconductance divided by the output conductance, or $g_{m} / g_{d s}$.
} 

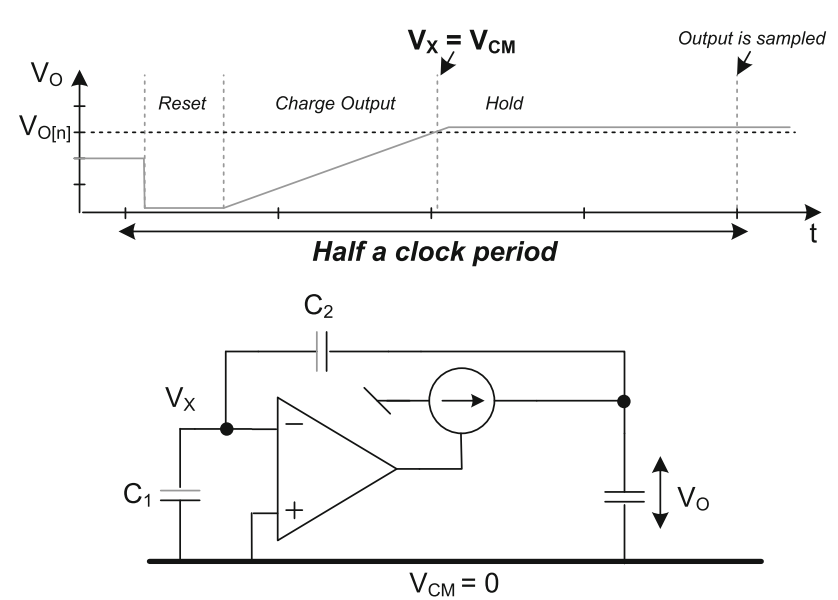

Fig. 1 Comparator-based switched-capacitor circuit. The sampling phase is equivalent to OTA-based switched-capacitor, and is not shown in the figure. At the start of the charge transfer phase the output is reset, after a delay the current source begins to charge the output. When a voltage of zero is detected between $V_{X}$ and $V_{C M}$ the current source is turned off

ensures that $V_{X}$ start below the virtual ground. The current source is turned on at the start of reset and use reset to settle. When reset ends the current source charges the output capacitance. The voltage at $V_{O}$ and $V_{X}$ rises until the comparator detects virtual ground $\left(V_{X}=V_{C M}=0\right)$, and turns off the current source.

\subsection{Sampling of stage output}

In our ADC the next stage samples at the end of the charge transfer phase. This is different from the original sampling in [1], where they used the signal from the comparator to sample in the next stage, thus ensuring no problems with charge leakage. The reason for our method was that we believed it would give us increased speed, we wanted our ADC to run at $100 \mathrm{MHz}$, not $10 \mathrm{MHz}$ as in [1], so we believed that it would be better to use the clock phases to sample, and not the comparator output. This was an error, and as a result we had to run the ADC above $10 \mathrm{MS} / \mathrm{s}$. Below $10 \mathrm{MS} / \mathrm{s}$ the change in voltage from the ramp stopped, to the output was sampled, became significant. Accordingly, we recommend that others use the comparator signal for sampling in the next stage, as suggested in [1].

\subsection{Comparator delay compensation}

Since any real comparator has a delay it takes a moment for the current source to turn off, which results in a overshoot of the output voltage. Methods to compensate for this overshoot exists, in $[1,3]$ they used a dual ramp system, first a fast ramp to estimate the output voltage, then a slow ramp to fine tune the output voltage. Since the ramp was slow the comparator delay caused an insignificant voltage change. A dual ramp system was also used in [4], but they included an additional compensation for overshoot using a switched-capacitor circuit. In [5] an analog signal was globally adjusted to compensate for the offset of the ADC. The scheme used in [6] (presented February 2009) is similar to the scheme we choose for our ADC, which was taped out July 2007, but we compensate for the comparator delay differently.

\subsection{Non-linearity caused by switches}

In an OTA based switched-capacitor circuit the voltages settle, and the current through the feedback network asymptotically approaches zero. In a CBSC circuit this does not happen, the current through the feedback network is almost constant until it is turned off. As a result, switch resistance causes an offset and a non-linearity [3, 5]. But effects can be minimized by splitting the current source [5], or reducing switch resistance. In our implementation lowthreshold voltage transistors were used in critical switches to get low resistance.

\section{The ADC architecture}

A system level diagram of the ADC is shown in Fig. 2. The ADC has seven 1.5-bit pipelined stages and a 1.5-bit flashADC. The target specification of the ADC was 10-bit, $100 \mathrm{MHz}$, and $10 \mathrm{~mW}$. So the ADC was designed as a 10bit ADC with eight 1.5-bit stages and a 2 bit flash-ADC. But measurements showed more noise than expected (the noise was dominated by the digital IO). To reduce the noise coupling we disconnected the output from the flash ADC, and turned off currents in Stage 8. The sub-ADC in Stage 8 was used for the LSB.

Bias current was controlled with a on-board variable resistor. External reference voltages were used to save design time, so the power consumed by the references is not included in reported power dissipation. The digital outputs from the SADCs are brought off-chip by CMOS logic IO buffers. Synchronization, recombination and digital error correction of the output bits was performed in software. An offline calibration algorithm, which will be explained later, was used to find the correct comparator offset correction and current source current. An on-chip non-overlapping clock generator made the clock phases needed by the pipelined stages.

\subsection{Pipelined stage}

The first stage (Stage 1) is shown in Fig. 3 during sampling and charge transfer. Stages 2-7 are identical to Stage 1 
Fig. 2 System level diagram of the pipelined ADC
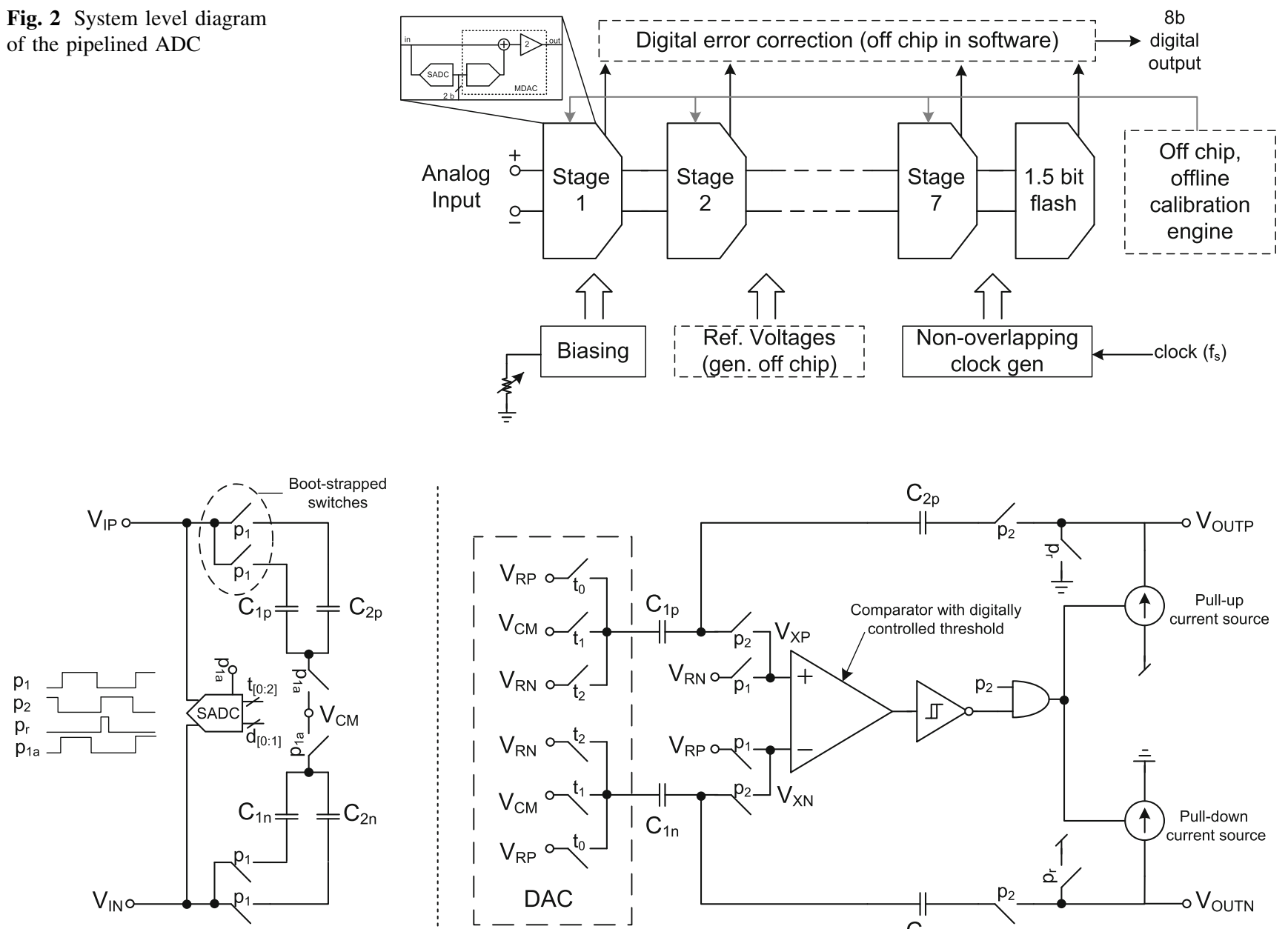

Sampling $\left(p_{1}\right)$

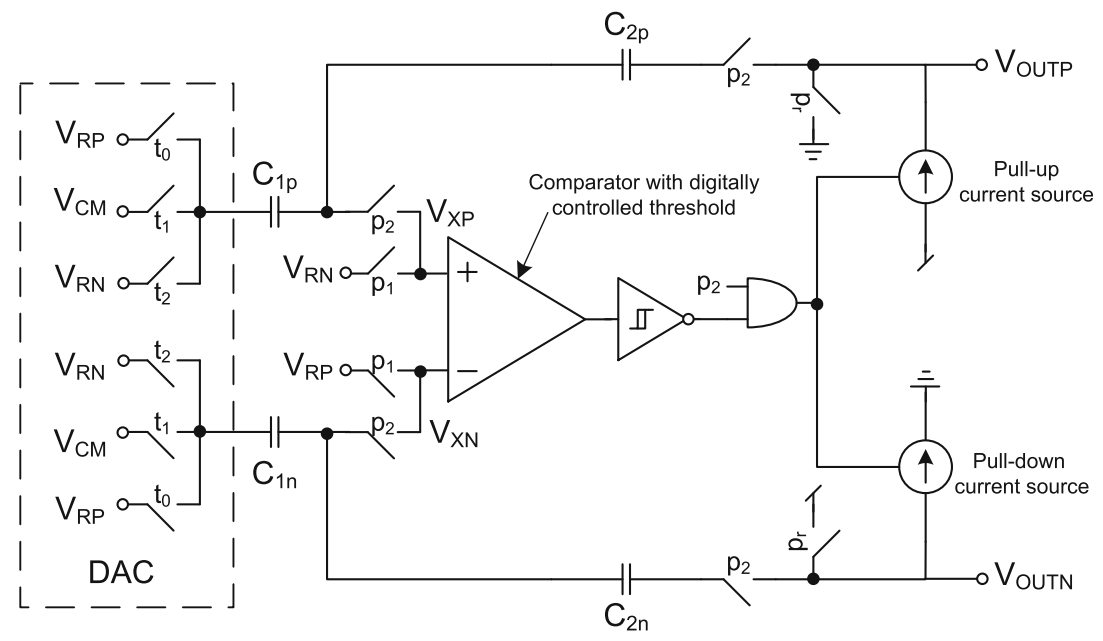

Charge transfer $\left(p_{2}\right)$

Fig. 3 Stage one shown during the two phases, sampling and charge transfer

with the exception of the input switches and capacitor size. The input switches are bootstrapped in the first stage, but regular transmission gates in later stages. Each pipelined stage has a 1.5-bit analog-to-digital converter (SADC). The signals $t_{0}, t_{1}$ and $t_{2}$ are the digital outputs of the SADC that controls the DAC during charge transfer. The stage operates on four clock phases: $p_{1}, p_{2}, p_{r}$ and $p_{1 a}$. An advanced (as in transitions before) clock phase $\left(p_{1 a}\right)$ samples the input signal before $p_{1}$ turns off, this reduces the problem of signal dependent charge injection from $p_{1}$ switches. The voltages $V_{C M}, V_{R N}, V_{R P}$ are the common mode voltage, negative reference voltage, and positive reference voltage, respectively.

\section{ADC components}

An ADC has several components, it has comparators, clock generators, digital IO, bias distribution and switches. In a
CBSC ADC the key components are the high outputresistance current sources and the comparator to turn them off. In this section we'll go through the key components.

\subsection{Current sources}

The current sources in a CBSC ADC must have high output resistance and they must tolerate high swing to get a linear voltage ramp. A standard current mirror in a $90 \mathrm{~nm} \mathrm{CMOS}$ technology has a low output resistance, on the order of $100 \mathrm{k} \Omega$. A cascode can increase the output resistance around 10 times, up to $1 \mathrm{M} \Omega$. To further increase the output resistance we used gain boosting, also called active cascode, or regulated cascode [7]. With a one stage amplifier used as a gain booster the output resistance can increase 10 times, up to $10 \mathrm{M} \Omega$. A simplified schematic of the pull-down current source can be seen in Fig. 4, for the actual schematics see [8]. 


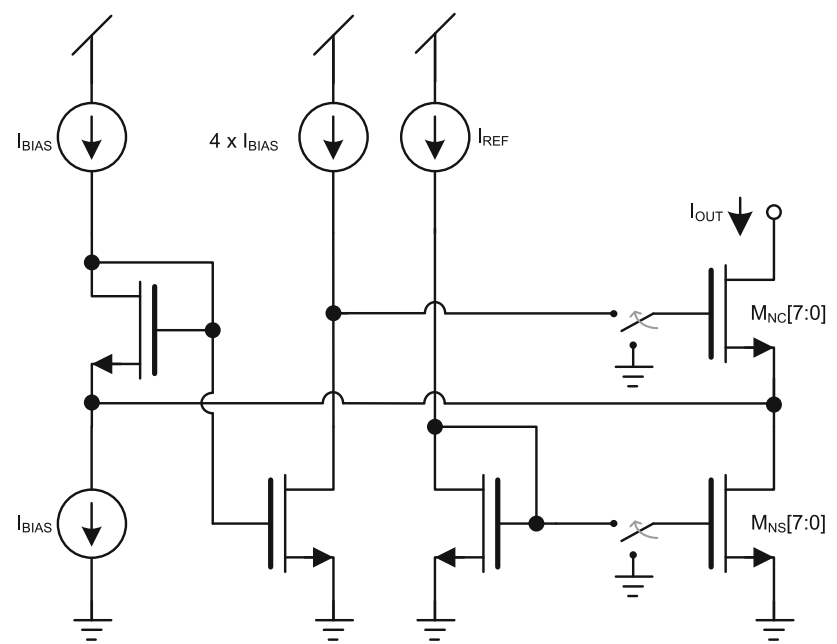

Fig. 4 NMOS current source. $M_{N X}[7: 0]$ is a notation for a digitally controlled transistor size. A transistor pair (for example $M_{N C}[0]$ and $\left.M_{N S}[0]\right)$ can be disabled by a calibration bit. The enabled transistor pairs gates are pulled to ground when the comparator output in Fig. 3 is high

The size of the cascode and current source transistor can be digitally controlled (transistor width $M[7: 0]$ ) by calibration bits. The current scaling is controlled by an 8-bit word. With this architecture the gain booster will see a varying load, but this was not a significant design challenge.

\subsubsection{Output common mode in CBSC circuits}

Assume that the current sources are symmetric, and that we reset $V_{\text {OUTN }}$ to VDD and $V_{\text {OUTP }}$ to VSS. When the comparator turns off the current sources the common mode output will be at VDD/2. A delta current between the pullup and pull-down current sources will change the output common mode voltage. The output voltage of the stage is given by $d V=\frac{I(t) \times d t}{C}$, where we assume $I(t)$ is constant. With an output capacitance of $600 \mathrm{fF}$, a delta current of $5 \mu \mathrm{A}$, and a charge-transfer phase of $8 \mathrm{~ns}$, the common mode will change $67 \mathrm{mV}$. This is within acceptable limits, so our circuit did not include any common mode feedback circuit. For a detailed discussion of CBSC output common mode and power supply rejection ratio see [9].

\subsection{Comparator with adjustable threshold}

A two-stage continuous-time amplifier with a differential first stage and single ended common source second stage was used as the comparator. A simplified schematic of the comparator is shown in Fig. 5.

In phase $p_{1}$ the comparator inputs are reset to $V_{R N}$ and $V_{R P}$ (as shown in Fig. 3), so the output is known at the end

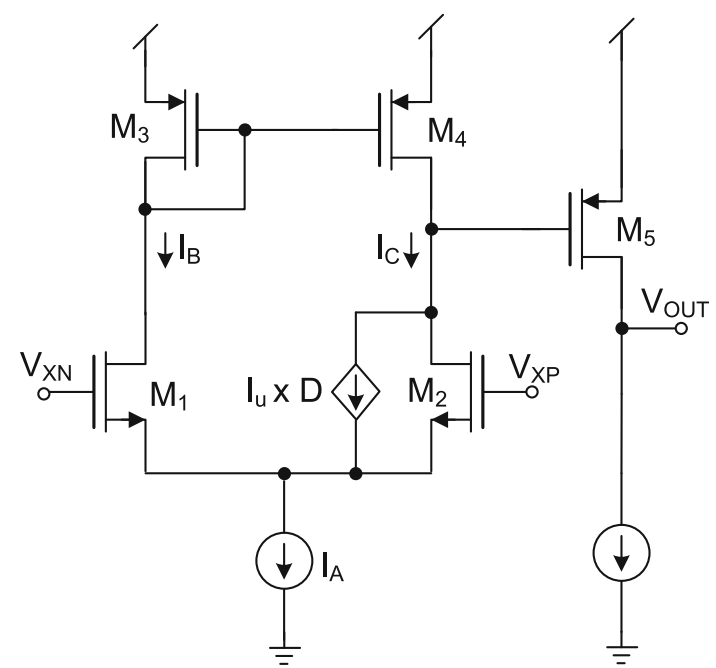

Fig. 5 Comparator with adjustable threshold

of sampling phase $\left(p_{1}\right)$. With this preset the control logic to turn off the current source at the right time consist of a single Schmitt trigger and an AND gate. The preset also ensures that the comparator does not need to slew when we enter the charge transfer phase $\left(p_{2}\right)$, where we have very little time to do anything more than strictly necessary.

In the charge transfer phase the stage outputs are reset $\left(p_{r}\right)$, forcing a slight increase in $V_{X N}$ and decrease in $V_{X P}$, the amount of change depends on the stage input voltage (see Fig. 6 for a visualization). When reset is complete $\left(p_{r}\right.$ goes low) the current sources charge the output, and $V_{X N}$ falls and $V_{X P}$ rises. When they meet we want to turn off the current sources, because that is when we have zero charge across capacitors $C_{1 p}$ and $C_{1 n}$ (assuming the DAC output is connected to $V_{C M}$ ), and all the charge is transferred to $C_{2 p}$ and $C_{2 n}$.

Figure 6(a)-(c) shows $V_{X N}$ and $V_{X P}$ as a function of time for different comparator thresholds $\left(V_{c t}\right)$. The comparator should turn off current sources when $V_{X P}=V_{X N}$, but because the comparator has a delay $\left(t_{c}\right)$ the current sources turn off later, causing an overshoot (Fig. 6(a)). Adjusting the threshold of the comparator changes the amount of overshoot. If $V_{c t}$ is adjusted optimally there is no overshoot (Fig. 6(b)). If $V_{c t}$ is lower than the optimal value the output undershoots (Fig. 6(c)). From the figure we see that a nonoptimal threshold cause an offset in the stage output, as shown in [10].

To control the comparator threshold we use a 6-bit current DAC in parallel with $M_{2}$, shown as a controlled source in Fig. 5. In the figure $I_{u}$ is a unit current and $D$ is an integer given by $D=2^{0} b_{0}+2^{1} b_{1}+2^{2} b_{2}+2^{3} b_{3}+2^{4} b_{4}+$ $2^{5} b_{5}$. The current in the current source $I_{A}$ is the sum of the two branch currents $\left(I_{A}=I_{B}+I_{C}\right)$. The comparator threshold is defined as the differential input voltage when 
Fig. 6 Voltage versus time for the nodes $V_{X N}$ and $V_{X P}$ as a function of comparator threshold. a Comparator threshold equal to zero. b Optimal comparator threshold. c Comparator threshold less than the optimal value (a)

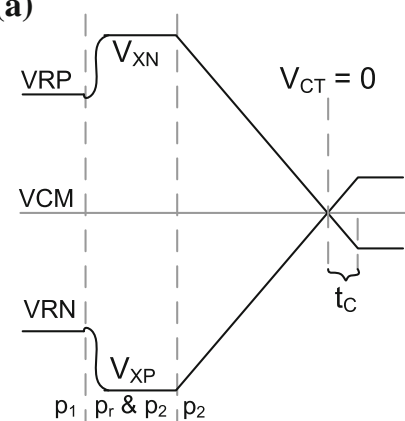

(b)

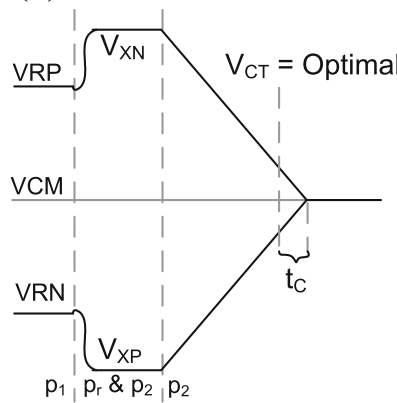

(c)

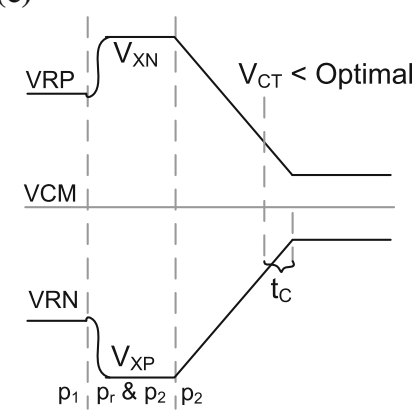

the branch currents are equal $\left(I_{B}=I_{C}\right)$. Equal currents occur when

$\beta V_{E F F, 1}^{2}=\beta V_{E F F, 2}^{2}+I_{u} \times D$

where $\beta=\frac{1}{2} \mu_{n} C_{o x} \frac{W}{L}$ and $V_{E F F, 112}$ is the effective gate overdrive of transistors $M_{1 \mid 2}{ }^{2}$ If $D=0$ the currents are equal when the effective gate overdrives are equal, which occurs when the inputs are equal. If $D>0$ the currents are equal when the effective overdrive of $M_{1}$ is larger than the effective overdrive of $M_{2}$, which occurs when $V_{I N}>V_{I P}$.

The nominal delay of the comparator (including Schmitt trigger and logic gates) is $t_{c}=0.5 \mathrm{~ns}$. With the 6-bit DAC the effective delay of the comparator can be controlled from $t_{c}=-0.9$ to $t_{c}=0.5 \mathrm{~ns}$.

\subsection{Bootstrapped switches}

The input switches in the first stage of a pipelined converter feel the full force of a sinusoidal input signal, which means that the voltage dependent switch resistance affects the linearity of the converter. To ensure high linearity the switch resistance must be low, but in nano-scale technologies the difference between the power supply and the sum of the threshold voltages is low (around $1.2 \mathrm{~V}-2 \times$ $0.5 \mathrm{~V}=0.2 \mathrm{~V}$ for an low-power $90 \mathrm{~nm}$ CMOS technology, compared to around $1.8 \mathrm{~V}-2 \times 0.5 \mathrm{~V}=0.8 \mathrm{~V}$ in an $0.18 \mu \mathrm{m}$ CMOS technology), so the overdrive of a transmission-gate is low, and the resistance high when the input signal is mid-rail. A transmission gate becomes prohibitively large at sufficiently low resistance. To reduce the switch size we can use a constant overdrive (constant $V_{g s}-V_{t h}=V_{\text {eff }}$ ), and to achieve a constant overdrive we can use bootstrapped switches. There are two basic types of bootstrapped switches, continuous time bootstrapped switches and switched-capacitor bootstrapped switches. Examples of the switched capacitor kind can be seen in [11]. We've used a type of continuous time bootstrapped

\footnotetext{
${ }^{2}$ Here we assume a square law model for the transistors, and that the transistors are in strong inversion and saturation
}

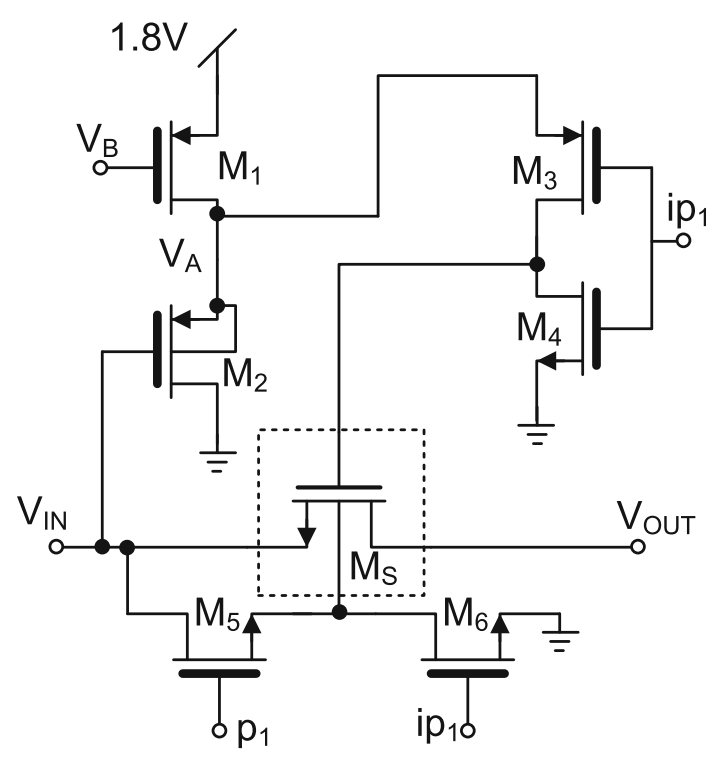

Fig. 7 Continuous time bootstrapped switch. The transistors with thick gates are thick oxide devices. The switch transistor $\left(M_{S}\right)$ is a thin oxide, low threshold voltage, device

switches [12]. In our implementation a source follower tracks the input signal, and the gate-source voltage of the source follower sets the gate-source voltage of the switch. Figure 7 shows the continuous time bootstrapped switch. We've modified the switch from [12] by adding $M_{3}$. Without $M_{3}$ the source of $M_{2}$ is reset to zero when we turn off the switch, and thus has to slew when we turn the switch on. By turning off the switch with $M_{3}$ and $M_{4}$ we get a faster turn-on time, because the source of $M_{2}$ is no longer reset to zero. To avoid reliability problems we've used thick oxide transistors in the switch. If we were to use standard oxide transistors the $V_{D S}$ of $M_{3}, M_{4}$ and $M_{2}$ would be too large. At high $V_{D S}$ the transistors can be damaged by hot-carrier injection [13]. Using thick oxide transistors removed this challenge, but for the switch transistor we've used a low-threshold thin oxide device to reduce the switch resistance. To reduce the switch resistance even more we connect the bulk to the source when the switch is on, removing the body effect. When the switch is off $M_{6}$ shorts 
the bulk to ground to avoid forward biasing the bulk-drain PN junction.

\subsection{Sub analog-to-digital converter}

The stage sub analog-to-digital converter (SADC) used dynamic comparators. The comparators in the SADC can have large offsets (up to $\pm 100 \mathrm{mV}$ in our implementation), because the pipelined architecture can correct the offset [14]. Since we can have large offsets we used a dynamic comparator referred to as a resistive divider [15].

\section{Test setup and calibration}

A custom PCB was made to test the ADC. The ADC needed a clock signal, an input signal, digital control signals, and references. The test setup can be seen in Fig. 8. We used two Rode \& Schwarz SML03 signal sources for the clock signal and high frequency input signal. To remove signal source harmonics we used fifth-order passive filters. The input signal was fed through a DC block capacitor, a $10 \mathrm{nH}$ inductor and a RF transformer (MiniCircuits ADT1-1WT) to do single ended to differential conversion. The common mode was set at VDD/2 with a resistor ladder. The resistors and capacitors before the ADC input form a low pass filter, with a cut off of around $40 \mathrm{MHz}$. To get fast clock edges a high speed comparator (MAX 961U) was used for sinusoid to square wave conversion. To power the chip we used low-dropout CMOS regulators (ADP1715), one regulator for each of the power domains. The PCB has five power domains, one analog $1.2 \mathrm{~V}$ supply, an analog $1.8 \mathrm{~V}$ supply, a $1.2 \mathrm{~V}$ digital supply for on-chip buffers, a $1.2 \mathrm{~V}$ digital supply for on-board output buffers, and a $3.3 \mathrm{~V}$ supply for clock generation and optocuplers.

One of the major limitations in the current ADC and PCB design is excessive coupling of noise from digital outputs. The on-chip CMOS IO buffers are fed directly from the internal SADCs. The outputs, running at $60 \mathrm{MS} / \mathrm{s}$, change value each half period, which generates significant on-board noise. Since the references for the ADC are onboard, and not on-chip they have limited noise immunity, and thus the ENOB suffers. To reduce the noise injection we reduced power supply of the digital buffers (on-board, and on-chip) to $0.85 \mathrm{~V}$, this limited our speed to $60 \mathrm{MS} / \mathrm{s}$, but allowed us to get an ENOB of 7.05-bit.

To calibrate the ADC we shift in a 272-bit calibration string. Each pipelined stage has 6-bits for the comparator, 8-bits per current source, 2-bits for additional current in

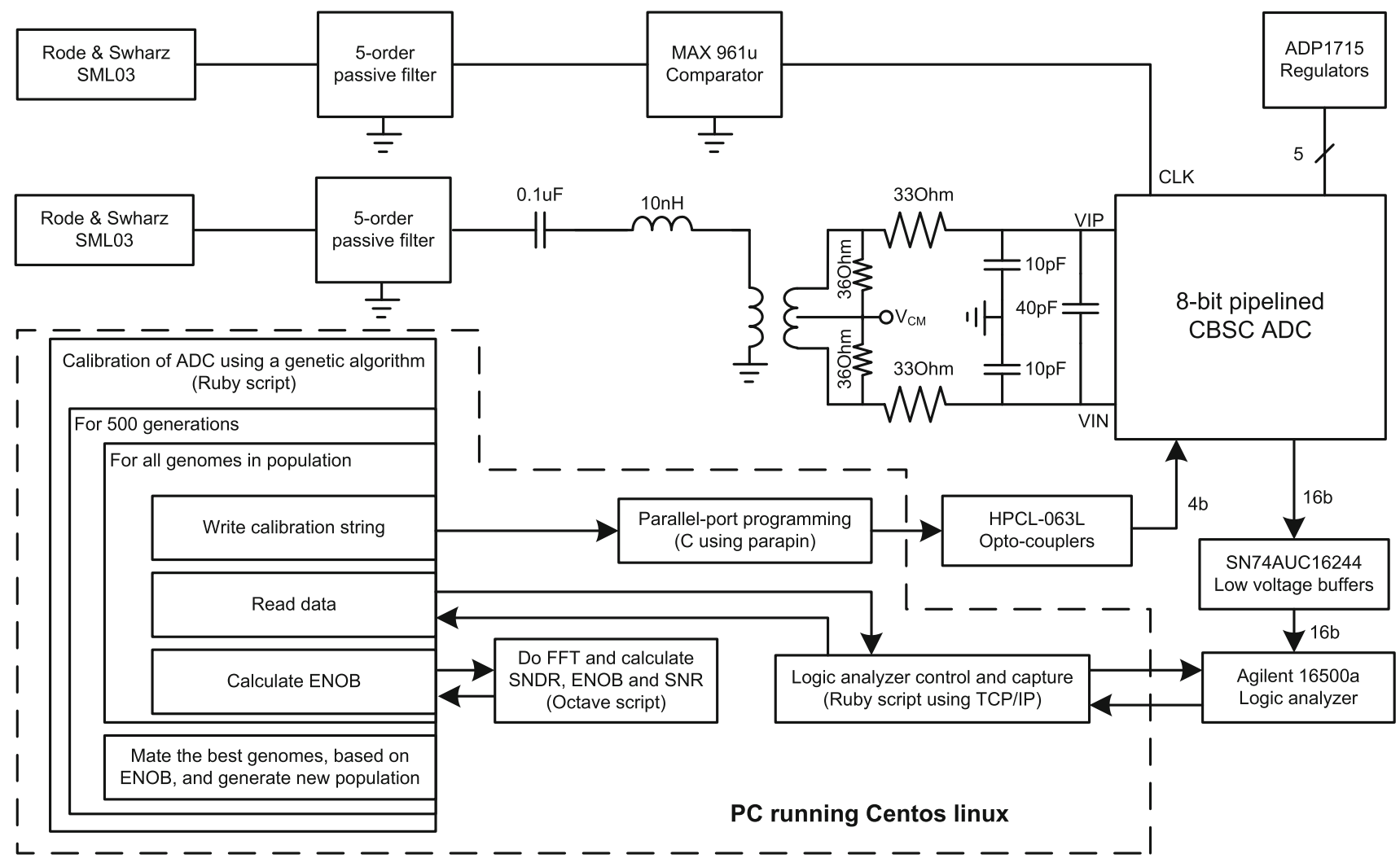

Fig. 8 ADC test setup 
Fig. 9 The ADC calibration string serial interface. The calibration string is shifted into a 272-bit register, and when the shift is complete the calibration string is loaded in parallel into a second register that is connected to the calibration lines for each stage

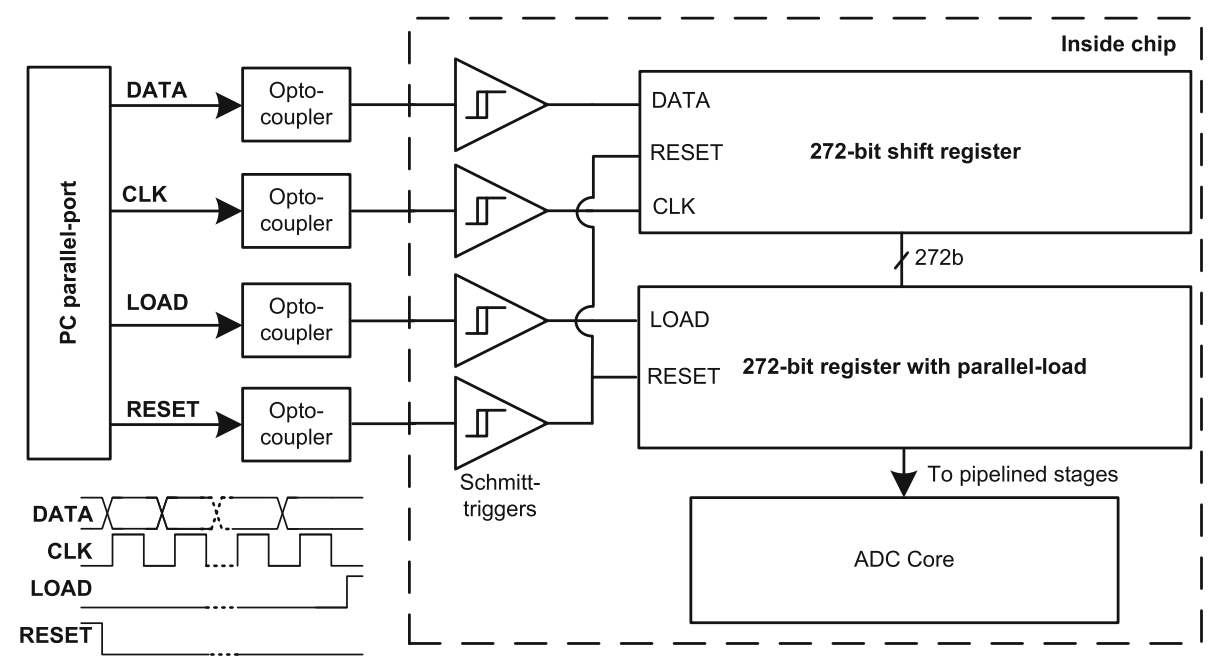

current source gain boosters, and 8-bits to control an in-stage analog test-mux, in total 32-bits per stage. There are 8-stages of 32-bits and a 16-bit string to control testmuxes, in total 272-bits. In the ADC we only use seven of the pipelined stages, so for calibration 154-bits are used. The calibration string is prepared in a Ruby script and passed to a C-program that writes the bits to the ADC with the PC parallel port (using the parapin library [16]) through optocouplers (HCPL-260L) into the serial interface of the ADC, shown in Fig. 9.

The output of the ADC (2-bits per stage times 7 -stages +1.5 -bit flash $=16$-bits) were buffered by low voltage buffers (SN74AUC16244) and captured by an Agilent 16500a logic analyzer. The data was read via an Ethernet connection to a PC, and post-processed there.

\subsection{Calibration of ADC}

The calibration algorithm described here is not suitable, nor is it intended, for a commercial ADC, it is too slow. The calibration algorithm is only suitable for lab testing to prove that there is a calibration setting that compensates for the comparator delay. We did not focus on implementing an efficient algorithm, our plan was to make the comparator and current sources digitally controllable, and prove that there is a calibration setting that can compensate for the comparator delay.

To find a suitable calibration string we used an algorithm inspired by evolution. The algorithm belongs to a class of algorithms often referred to as a "genetic algorithms" [17]. We start by generating 30 calibration strings (individuals), which make up our first population. The initial population was based on a solution found by hand tuning. Each of the individuals were tested in the ADC, and the effective number of bits (ENOB) was calculated.
During this test the ADC had an input signal of $29.4 \mathrm{MHz}$. Each of the individuals in the population were ranked according to their ENOB. The two best individuals were transferred to the new population directly. The rest of the new population was filled by mating the best individuals from the previous population. Each individual undergoes mutation after mating, and the site of mutation is random (for example bit one of the six-bit comparator string in pipelined stage four switches from 0 to 1). Mutation ensures that the algorithm does not get stuck with similar individuals in the whole population. For each new population (generation) we repeat the process, and this goes on for five hundred generations (roughly 15,000 calibration strings tested), which takes around $7 \mathrm{~h}$. As time progresses each generation improves, and the population converges towards a solution. An example of a calibration run can be seen in Fig. 10. The starting point for the calibration was a hand calibrated ENOB of 5.8-bit. The best solution gave an ENOB of 7.05-bit at $60 \mathrm{MS} / \mathrm{s}$. The best calibration string was used for all measurements in the next section.

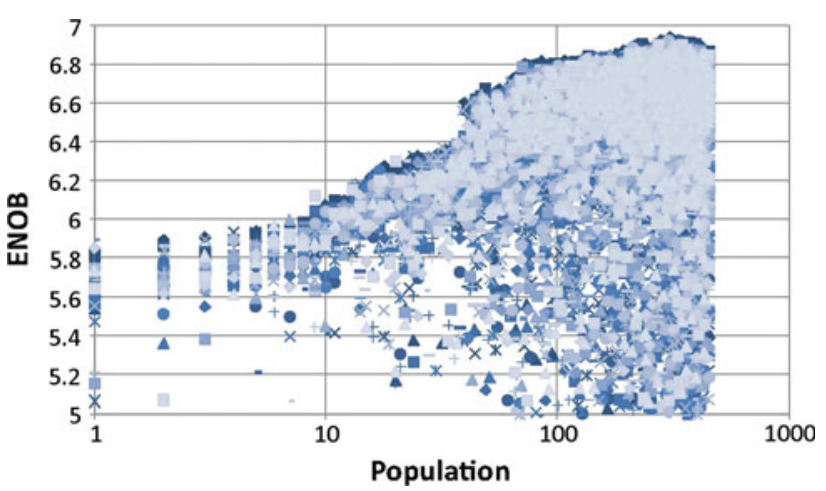

Fig. 10 Evolution of the ENOB for a calibration run, initial solution was 5.8-bit ENOB 
(a)
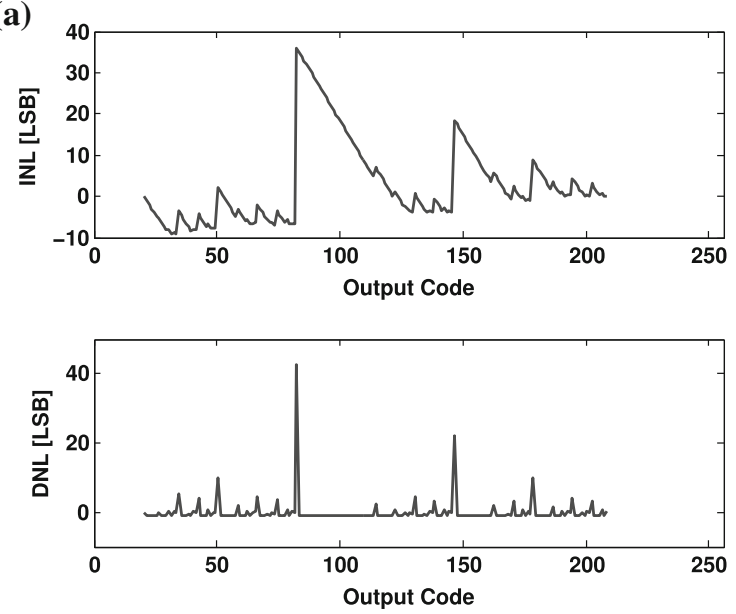

(b) 2
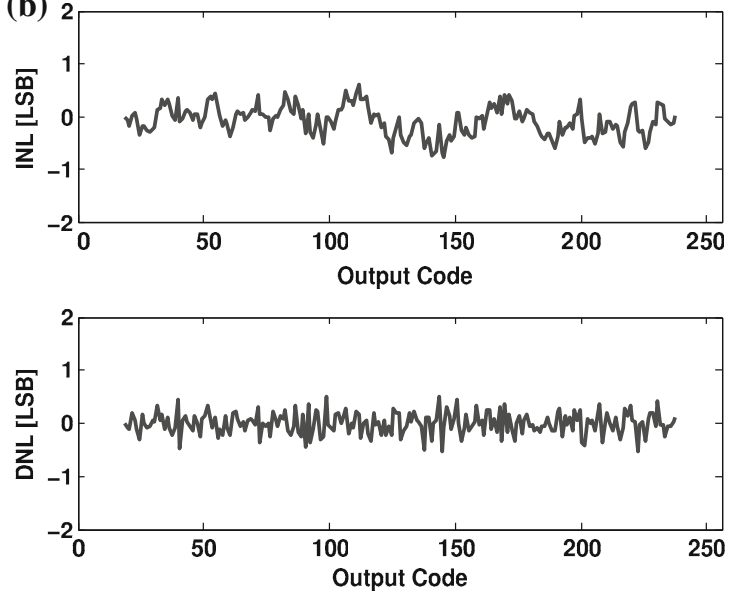

Fig. 11 INL and DNL for uncalibrated, and calibrated ADC. a No calibration, default values set before production. b After calibration of comparator offset and current source current

Table 1 Summary of calibrated ADC performance

\begin{tabular}{ll}
\hline Technology & $1.2 \mathrm{~V} / 1.8 \mathrm{~V} 90 \mathrm{~nm}$ CMOS \\
Sampling frequency & $60 \mathrm{MS} / \mathrm{s}$ \\
Resolution & $8 \mathrm{bits}$ \\
Full scale input & $0.8 \mathrm{~V}$ \\
Area & $0.85 \mathrm{~mm} \times 0.35 \mathrm{~mm}$ \\
DNL (LSB) & $0.52 /-0.54$ \\
INL (LSB) & $0.6 /-0.77$ \\
SNR (29.4 MHz input) & $44.5 \mathrm{~dB}$ \\
SNDR (29.4 MHz input) & $44.2 \mathrm{~dB}$ \\
SFDR (29.4 MHz input) & $60 \mathrm{~dB}$ \\
ADC core power & $5.9 \mathrm{~mW}$ \\
Clock power & $2.3 \mathrm{~mW}$ \\
Input switches $(1.8 \mathrm{~V})$ & $0.3 \mathrm{~mW}$ \\
\hline
\end{tabular}

\section{Measurement results}

The essence of this paper is the digitally controlled comparator threshold that compensates for the comparator delay. The development of an efficient calibration algorithm has been left for future research.

The default comparator threshold and current source current set before production caused an excessive overshoot, as a result the maximum integral non-linearity (INL) is 36 LSB (seen in Fig. 11(a)), and the ADC has an ENOB of 2.5-bit. After calibration, with optimal comparator threshold and current source current the ADC has an ENOB of 7.05-bit and a maximum INL of 0.7 LSB. This demonstrates that the variations caused by processing can be canceled by digitally adjusting the threshold of the comparator.

A summary of the ADC performance is shown in Table 1. It achieves a signal-to-noise and distortion ratio (SNDR) of 44.2-dB (7.05-bit) with a sampling frequency

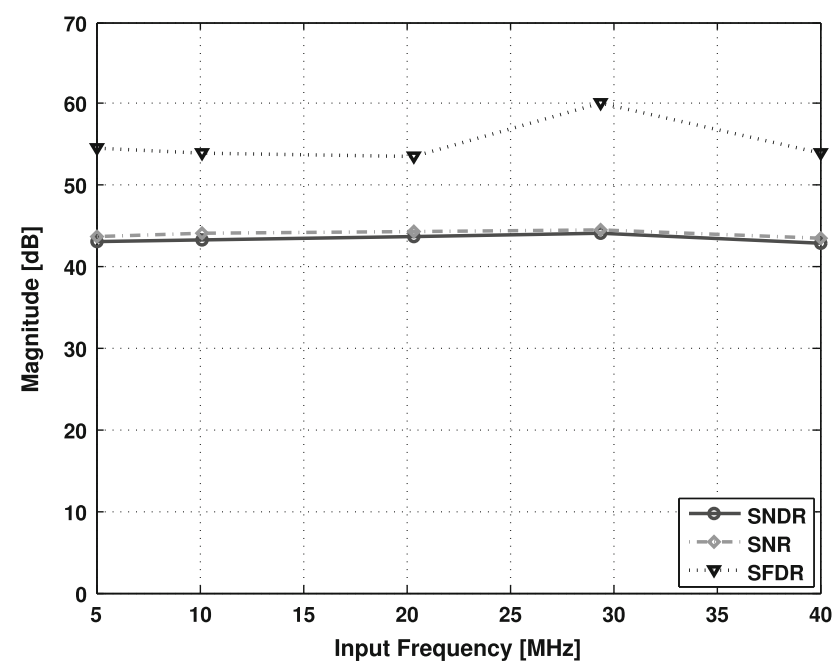

Fig. 12 SNDR, SNR and SFDR versus frequency, sampling frequency is $60 \mathrm{MS} / \mathrm{s}$

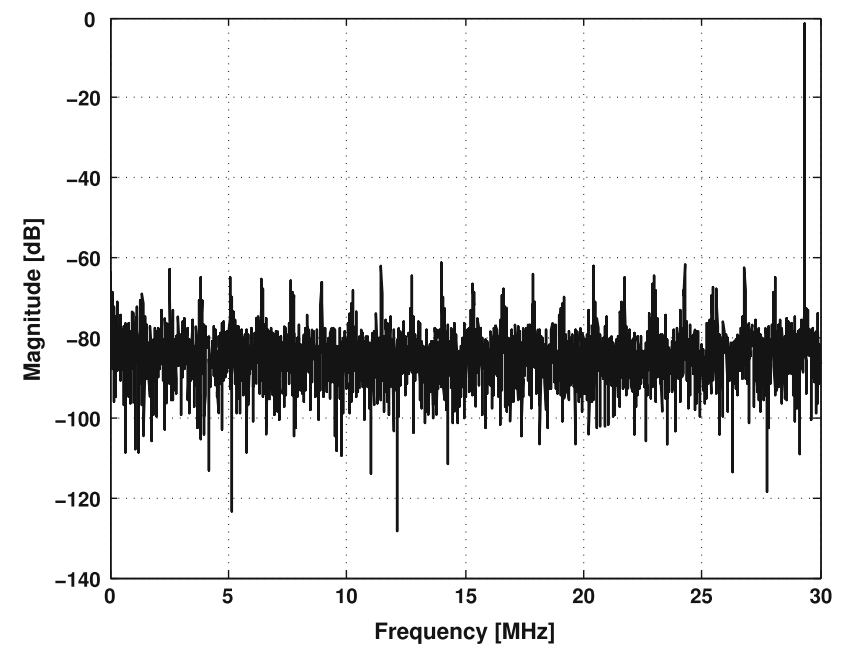

Fig. 13 A 8192 point FFT of the ADC output 
Table 2 Comparison to state-of-the-art

\begin{tabular}{|c|c|c|c|c|c|c|c|}
\hline $\begin{array}{l}\text { Process } \\
(\mathrm{nm})\end{array}$ & $\begin{array}{l}\text { Power } \\
(\mathrm{mW})\end{array}$ & $\begin{array}{l}\text { ENOB @ } \\
\text { Nyquist bits }\end{array}$ & $\begin{array}{l}\text { Sampling } \\
\text { rate }(\mathrm{MS} / \mathrm{s})\end{array}$ & $\begin{array}{l}\text { Thermal FOM } \\
\text { (fJ/step) }\end{array}$ & $\begin{array}{l}\text { Walden FOM } \\
(\mathrm{pJ} / \mathrm{step})\end{array}$ & Architecture & Reference \\
\hline 90 & 70 & 6.56 & 770 & 10.1 & 0.96 & Sub-ranging & {$[20]$} \\
\hline 90 & 34 & $7.0 ?$ & 300 & 6.9 & 0.89 & Sub-ranging & [19] \\
\hline 90 & 2.6 & 6.85 & 10 & 19.5 & 2.17 & Pipelined & {$[21]$} \\
\hline 130 & 21 & 7.60 & 125 & 4.5 & 0.86 & Sub-ranging & {$[22]$} \\
\hline 180 & 30 & 7.68 & 200 & 3.5 & 0.73 & Pipelined & {$[23]$} \\
\hline 180 & 8.5 & 6.40 & 200 & 6.0 & 0.5 & Pipelined & {$[5]$} \\
\hline 90 & 8.5 & 7.05 & 60 & 8.1 & 1.07 & Pipelined & This work \\
\hline
\end{tabular}

$\left(f_{s}\right)$ of $60 \mathrm{MS} / \mathrm{s}$, an input signal of $f_{s} / 2$, and a power dissipation of $8.5 \mathrm{~mW}(5.9 \mathrm{~mW}$ for ADC core, $2.3 \mathrm{~mW}$ for clock generation and distribution, and $0.3 \mathrm{~mW}$ for input switches). An input signal amplitude of $-1 \mathrm{dBFS}$ was used during measurement. The ADC has a spurious free dynamic range (SFDR) of $60-\mathrm{dB}$. The SNDR and SNR change little with input frequency, and the effective resolution bandwidth extend well beyond $f_{s} / 2$ (as seen in Fig. 12).

A 8192 point FFT of the ADC output is shown in Fig. 13. Coherent sampling and a Hanning window was used to avoid spectral leakage.

\subsection{Comparison to state-of-the-art}

A comparison of our converter to other 8-bit ADCs with sample rate above $1 \mathrm{MS} / \mathrm{s}$ is shown in Table 2. One of the figure of merits used is the Thermal FOM [13], which can be calculated from the equation

$\mathrm{FOM}=\frac{\text { Power dissipation }}{2^{2 \times \mathrm{ENOB}} \times \text { Sampling frequency }}$,

which differs from the more conventional Walden FOM of

$\mathrm{FOM}=\frac{\text { Power dissipation }}{2^{\mathrm{ENOB}} \times \text { Sampling frequency }}$.

The Thermal FOM is more correct when comparing ADCs with different ENOB [18].

From the Thermal FOM in Table 2 we can see that the performance of our converter is good compared to other 8-bit converters in $90 \mathrm{~nm}$, only beaten by [19].

\section{Conclusion}

We presented a differential comparator-based switchedcapacitor (CBSC) pipelined ADC with comparator preset, and comparator delay compensation. Compensating for the comparator delay by digitally adjusting the comparator threshold improves the ADC resolution from 2.5-bit to 7.05-bit. The ADC was manufactured in a $90 \mathrm{~nm}$ CMOS technology, with a core area of $0.85 \mathrm{~mm} \times 0.35 \mathrm{~mm}$, a
$1.2 \mathrm{~V}$ supply for the core and $1.8 \mathrm{~V}$ for the input switches. It has an ENOB of 7.05-bit, and a power dissipation of $8.5 \mathrm{~mW}$ at $60 \mathrm{MS} / \mathrm{s}$.

Acknowledgments We thank Professors David Johns and Ken Martin for their advice during design of the ADC, and thank the Electrical and Computer Engineering Department, University of Toronto for their hospitality during our stay. We thank Johnny Bjørnsen for help with measurements.

Open Access This article is distributed under the terms of the Creative Commons Attribution Noncommercial License which permits any noncommercial use, distribution, and reproduction in any medium, provided the original author(s) and source are credited.

\section{References}

1. Sepke, T., Fiorenza, J. K., Sodini, C. G., Holloway, P., \& Lee, H.-S. (2006). Comparator-based switched-capacitor circuits for scaled CMOS technologies. In ISSCC digest of technical papers 2006 (pp. 220-221).

2. Lee, H.-S., Brooks, L., \& Sodini, C. G. (2010). Zero-crossingbased ultra-low-power A/D converters. Proceedings of the IEEE, 98(2), 315-332.

3. Fiorenza, J. K., Sepke, T., Holloway, P., Sodini, C. G., \& Lee, H.-S. (2006). Comparator-based switched-capacitor circuits for scaled CMOS technologies. IEEE Journal of Solid-State Circuits, 41(12), 2658-2668.

4. Shin, S.-K., You, Y.-S., Lee, S.-H., Moon, K.-H., Kim, J.-W., Brooks, L., et al. (2008). A fully-differential zero-crossing-based $1.2 \mathrm{~V} 10 \mathrm{~b} 26 \mathrm{MS} / \mathrm{s}$ pipelined ADC in $65 \mathrm{~nm}$ CMOS. In 2008 IEEE symposium on VLSI circuits, 2008, pp. 218-219.

5. Brooks, L., \& Lee, H.-S. (2007). A zero-crossing-based 8-bit $200 \mathrm{MS} / \mathrm{s}$ pipelined ADC. IEEE Journal of Solid-State Circuits, 42(12), 2677-2687.

6. Brooks, L., \& Lee, H.-S. (2009). A 12 b 50 MS/s fully differential zero-crossing-based ADC without CMFB. In ISSCC digest of technical papers, pp. 166-167.

7. Johns, D., \& Martin, K. (1997). Analog integrated circuit design. Wiley, New York.

8. Wulff, C., http://www.wulff.no/carsten.

9. Brooks, L., \& Lee, H.-S. (2009). A 12b, 50 MS/s, fully differential zero-crossing based pipelined ADC. IEEE Journal of SolidState Circuits, 44(12), 3329-3343.

10. Wulff, C. (2008). Efficient ADCs for nano-scale CMOS technology. Ph.D. dissertation, Norwegian University of Science and Technology, 2008. 
11. Steensgaard, J. (1999). Bootstrapped low-voltage analog switches. IEEE International Symposium on Circuits and Systems, 2, 29-32.

12. Jakonis, D., \& Svensson, C. (2002). A $1 \mathrm{GHz}$ linearized CMOS track-and-hold circuit. Proceedings of ISCAS, 5, V-577-V-580.

13. Lewyn, L., Ytterdal, T., Wulff, C., \& Martin, K. (2009). Analog circuit design in nanoscale cmos technologies. Proceedings of the IEEE, 97(10), 1687-1714.

14. Cho, T. B., \& Gray, P. R. (1995). A 10b, 20 msample/s, $35 \mathrm{mw}$ pipeline A/D converter. IEEE Journal of Solid-State Circuits, 30(3), 166-172.

15. Sumanen, L., Waltari, M., \& Halonen, K. (2000). A mismatch insensitive CMOS dynamic comparator for pipeline A/D converters. In Proceedings of ICECS (Vol. 1, pp. 32-35).

16. Parapin, http://parapin.sourceforge.net/.

17. Back, T., Hammel, U., \& Schwefel, H.-P. (1997). Evolutionary computation: comments on the history and current state. IEEE Transactions on Evolutionary Computation, 1, 3-17.

18. Lee, H. S., \& Sodini, C. G. (2008). Analog-to-digital converters: Digitzing the analog world. Proceedings of IEEE, 96(2), 323-334.

19. Shimizu, Y., Murayama, S., Kudoh, K., \& Yatsuda, H. (2008). A split-load interpolation-amplifier-array $300 \mathrm{MS} / \mathrm{s} 8 \mathrm{~b}$ subranging ADC in $90 \mathrm{~nm}$ cmos. In Proceedings of ISSCC digest of technical papers, Feb 2008 (pp. 552-635).

20. Ohhata, K., Uchino, K., Shimizu, Y., Oyama, K., \& Yamashita, K. (2009). Design of a 770-MHz, 70-mW, 8-bit subranging ADC using reference voltage precharging architecture. IEEE Journal of Solid-State Circuits, 44(11), 2881-2890.

21. Shen, J., \& Kinget, P. R. (2008). A 0.5-V 8-bit 10-Ms/s pipelined ADC in 90-nm CMOS. IEEE Journal of Solid-State Circuits, 43(4), 787-795.

22. Mulder, J., Ward, C. M., Lin, C.-H., Kruse, D., Westra, J. R., Lugthart, M., et al. (2004). A 21-mW 8-b 125-MSample/s ADC in $0.09-\mathrm{mm}^{2} 0.13-\mu \mathrm{m}$ CMOS. IEEE Journal of Solid-State Circuits, 39(12), 2121-2133.

23. Kim, H.-C., Jeong, D.-K., \& Kim, W. (2005). A $30 \mathrm{~mW} \mathrm{8b}$ $200 \mathrm{MS} / \mathrm{s}$ pipelined CMOS ADC using a Switched-Opamp technique. In ISSCC digest of technical papers (pp. 284-285).

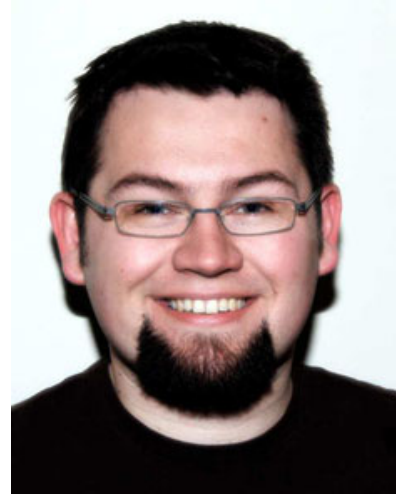

Carsten Wulff received the M.Sc. and Ph.D. degrees in Electrical Engineering from the Department of Electronics and Telecommunication, Norwegian University of Science and Technology (NTNU), in 2002 and 2008, respectively. During his Ph.D. work at NTNU, he worked on open-loop sigma-delta modulators and analog-to- digital converters in nanoscale CMOS technologies. In 2006-2007, he was a Visiting Researcher with the Department of Electrical and Computer Engineering, University of Toronto, Toronto, ON, Canada. $\mathrm{He}$ is currently a Research and Development Engineer with Nordic Semiconductor ASA, Trondheim, Norway. His present research interests includes analog and mixed-signal CMOS design and design of high-efficiency analog-to-digital converters.

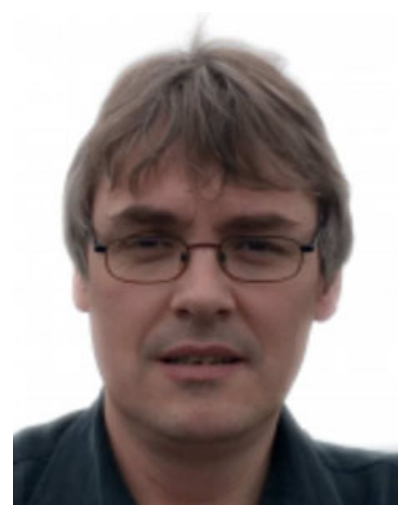

Trond Ytterdal received the M.Sc. and Ph.D. degrees in Electrical Engineering from the Norwegian Institute of Technology in 1990 and 1995, respectively. He was a Research Associate with the Department of Electrical Engineering, University of Virginia (1995-1996), and as a Research scientist with the Electrical, Computer and Systems Engineering Department, Rensselaer Polytechnic Institute, Troy, NY (19961997). From 1997 to 2001, he was a Senior ASIC Designer with Nordic Semiconductor, Trondheim, Norway. In 2001, he became a Professor at the Department of Electronics and Telecommunication, Norwegian University of Science and Technology (NTNU). His present research interests include design of analog integrated circuits, behavioral modeling and simulation of mixed-signal systems, modeling of nanoscale transistors, and novel device structures for application in circuit simulators. He has published more than 130 scientific papers in international journals and conference proceedings. He is a Coauthor of Semiconductor Device Modeling for VLSI (Englewood Cliffs, NJ: Prentice-Hall, 1993), Introduction to Device Modeling and Circuit Simulation (New York: Wiley, 1998), and Device Modeling for Analog and RF CMOS Circuit Design (New York: Wiley, 2003) and has contributed to several other books published internationally. He is also a Codeveloper of the circuit simulator AIM-Spice. Prof. Ytterdal is a member of The Norwegian Academy of Technological Sciences. 